\title{
Evaluación del Conocimiento Anátomo-Quirúrgico del Sistema de la Vena Cava Inferior por Médicos Residentes de Ciru- gía General
}

\author{
Evaluation of Anatomical and Surgical Understanding of th \\ Inferior Vena Cava System by General Surgery Interns
}

Rubén D. Algieri*; María S. Ferrante**; Cristian Flores ${ }^{* * *}$; Paulo Paglilla***; Humberto Insfran ${ }^{* * * *} \&$ Nicolás Ernesto Ottone ${ }^{* * * *, * * * * * * *}$

ALGIERI, R. D.; FERRANTE, M. S.; FLORES, C.; PAGLILlA, P.; INSFRAN, H. \& OTTONE, N. E. Evaluación del conocimiento anátomo-quirúrgico del sistema de la vena cava inferior por médicos residentes de cirugía general. Int. J. Morphol., 34(2):424-430, 2016.

RESUMEN: Las lesiones de vena cava inferior (VCI) han sido descritas como la lesión vascular abdominal mas frecuente encontradas en los centros de trauma. Puede lesionarse entre la supra e infrarrenal; y en el caso de lesión retrohepática, se asocia con alta mortalidad. En heridas penetrantes conllevan una elevada mortalidad. Hasta un $50 \%$ de los individuos que experimentan estas lesiones muere antes de llegar al hospital; y la tasa de mortalidad entre los que llegan con vida a un centro de trauma varía entre $20-57 \%$. Se evaluaron a 12 cirujanos en formación, respecto de los conocimientos anatómicos del sistema de la VCI y sus relaciones, con material cadavérico formalizado mediante listas de verificación (Abril/2014). Luego de un período de entrenamiento mediante la disección anatómica de 10 cadáveres formolizados (Mayo/2014-Agosto/2014); se realizó una nueva evaluación de los médicos residentes de cirugía general en formación mediante listas de verificación, con material cadavérico fresco (Septiembre/2014). Durante la primera evaluación la vena iliaca común derecha e izquierda, fueron identificadas por $8(66,66 \%)$ residentes. Siete $(58,33 \%)$ mencionaron los segmentos de la VCI en intratorácico y abdominal. Seis $(85,71 \%)$ mencionaron la porción intrapericárdica y uno $(14,29 \%)$ la porción extrapericárdica. La porción o segmento subduodenal fue identificado como subhepático por siete $(58,33 \%)$ residentes; y solo dos $(16,66 \%)$ mencionaron los segmentos subduodenal, retroduodenopancreático y supraduodenopancreático como parte del segmento subhepático. Tres (25\%) no mencionaron los diferentes segmentos de la VCI (abdominal). El segmento retrohepático fue identificado por ocho $(66,66 \%)$. Durante la segunda evaluación la vena iliaca común derecha e izquierda, fueron identificadas por todos los residentes. Once $(91,66 \%)$ mencionaron los segmentos de la VCI en intratorácico y abdominal, además de la porción intrapericárdica y la porción extrapericárdica. El segmento subduodenal fue identificado como subhepático por dos (16,66 \%) residentes; y nueve (75\%) mencionaron los segmentos subduodenal, retroduodenopancreático y supraduodenopancreático como parte del segmento subhepático. El segmento retrohepático fue identificado por once $(91,66 \%)$ residentes. La observación y disección anatómica de la VCI en material cadavérico durante la formación del cirujano general, brinda un importante método de enseñanza, capacitación y entrenamiento hacia el reconocimiento de las diferentes estructuras anatómicas de la región, pudiendo luego ser aplicado en cirugía. La metodología de supervisión y evaluación mediante listas de verificación, por médicos especialistas, es una opción a agregar a los programas de formación para mejorar el proceso de educación.

PALABRAS CLAVE: Vena cava inferior; Modelos cadavéricos; Conocimiento anátomo-quirúrgico; Entrenamiento quirúrgico; Cirujanos en formación.

\section{INTRODUCCIÓN}

Las heridas penetrantes abdominales son la causa más común de lesiones vasculares a este nivel; representan entre 90 y $95 \%$ de todas las lesiones vasculares intraabdominales.
El traumatismo abdominal cerrado es responsable aproximadamente del 5 al $10 \%$ restante (Feliciano, 1996; Feliciano et al., 1999; Asencio et al., 2007). De todos los pacientes

Especialista en Cirugía General. Jefe de Servicio de Cirugía General del Hospital Aeronáutico Central. Prof. Regular Adjunto de Anatomía (Facultad de Medicina - UBA). Docente Adscripto en Cirugía, Universidad de Buenos Aires, Buenos Aires, Argentina.

** Especialista en Cirugía General. Instructora de Residentes de Cirugía General del Hospital Aeronáutico Central. Prof. Jefe de Trabajos Prácticos de Anatomía (Facultad de Medicina - UBA), Universidad de Buenos Aires, Buenos Aires, Argentina.

**** Residente de Cirugía General del Hospital Aeronáutico Central. Buenos Aires, Argentina.

***** Centro de Investigación en Ciencias Odontológicas (CICO), Facultad de Odontología, Universidad de La Frontera, Temuco, Chile.

****** Programa de Doctorado en Ciencias Morfológicas, Facultad de Medicina, Universidad de La Frontera, Temuco, Chile. 
sometidos a laparotomía por heridas abdominales por arma de fuego, cerca de $25 \%$ presentan lesiones vasculares, mientras que sólo $10 \%$ de aquellos intervenidos por heridas abdominales por arma blanca, evidencian lesión vascular (Feliciano; Feliciano et al.; Asencio et al.).

Las lesiones abdominales penetrantes son normalmente debidas a heridas por arma blanca o arma de fuego; las contusiones abdominales son el resultado de accidentes de tráfico con traumatismo directo sobre el abdomen (Asencio et al.).

Las lesiones de vena cava inferior (VCI) han sido descritas como la lesión vascular abdominal mas frecuente encontradas en los centros de trauma. Las lesiones penetrantes de VCI ocurren en un 4,7\% de los traumatismos abdominales (Fuentes et al., 2003). Puede lesionarse entre la supra e infrarrenal; y en el caso de lesión retrohepática, se asocia con alta mortalidad (3). Así, las heridas penetrantes de VCI conllevan una mortalidad de alrededor del $50 \%$ influenciada por la exsanguinación, acidosis metabólica, hipotermia y a la falta de prelación en su manejo (Fuentes et al.). Hasta un $50 \%$ de los individuos que experimentan estas lesiones muere antes de llegar al hospital; y la tasa de mortalidad entre los que llegan con vida a un centro de trauma varía entre 20 a 57 \% (Ponce de León Espindola \& López Cueto, 2011).

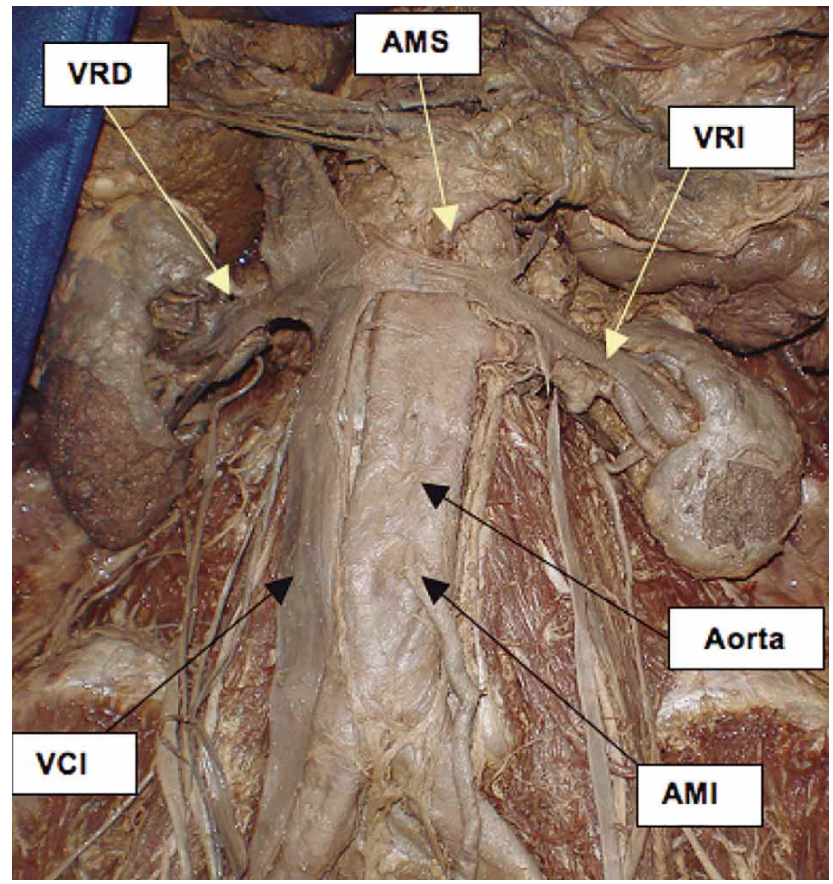

Fig. 1. Material cadavérico formolizado al $10 \%$, utilizado para priemera evaluación. $\mathrm{VCI}=$ vena cava inferior; $\mathrm{AMI}=$ arteria mesentérica inferior; VRI= vena renal izquierda; $\mathrm{VRD}=$ vena renal derecha; $\mathrm{AMS}=$ arteria mesentérica superior.
En la bibliografía anatómica se describe a la VCI en dos porciones, intraabdominal e intratorácica (Testut \& Latarjet, 1943; Latarjet \& Ruiz Liard, 2005; Rouviere \& Delmas, 2005; Moore et al., 2013; Pró, 2014); y algunos autores describen segmentos para cada una de estas porciones (Latarjet \& Ruiz Liard; Pró); lo que podría aplicarse en la cirugía abdominal y particularmente en trauma. Por lo que es fundamental un adecuado conocimiento anatómico de la VCI y sus relaciones anatómicas, siendo la base esencial tanto para la sospecha lesional como para la realización de maniobras precisas con posterior control vascular en un paciente con riesgo de vida por lesión del sistema de la VCI.

El objetivo de este trabajo fue demostrar la necesidad del entrenamiento a médicos residentes de cirugía general mediante la utilización de modelos cadavéricos para la adquisición de conocimientos básicos anatomo-quirúrgicos del sistema de la vena cava inferior y sus relaciones anatómicas durante su formación.

\section{MATERIAL Y MÉTODO}

Se evaluó a 12 cirujanos en formación, respecto de los conocimientos anatómicos del sistema de la vena cava inferior y sus relaciones, con material cadavérico formalizado al $10 \%$ (Fig. 1), mediante listas de verificación (Abril/ 2014) (Fig. 2) (Tabla I). Luego de un período de entrenamiento mediante la disección anatómica de 10 cadáveres formolizados al 10\% (Mayo/2014-Agosto 2014); se realizó una nueva evaluación de los médicos residentes de cirugía general en formación mediante listas de verificación (Tabla I), con material cadavérico fresco (Fig. 2A-D) (Septiembre/2014).

\section{RESULTADOS}

Durante la primera evaluación la vena iliaca común derecha e izquierda, fueron identificadas por ocho residentes $(66,66 \%)$. Siete residentes $(58,33 \%)$ mencionaron los segmentos de la VCI en intratorácico y abdominal. Entre los siete que identificaron el segmento intratorácico, seis residentes $(85,71 \%)$ mencionaron la porción intrapericárdica y uno $(14,29 \%)$ la porción extrapericárdica.

El segmento abdominal fue identificado por 12 residentes $(100 \%)$. De éste segmento, la porción o segmento subduodenal fue identificado como subhepático por siete residentes $(58,33 \%)$; y solo dos $(16,66 \%)$ mencionaron los segmentos subduodenal, retroduodenopancreático y 
Tabla I. Lista de verificación utilizada para evaluación con material cadavérico formalizado al $10 \%$ y luego con material cadavérico fresco.

\begin{tabular}{lll}
\hline Estructuras a identificar & NO \\
\hline
\end{tabular}

Vena iliaca común derecha

Vena ilíaca común izquierda

Vena cava inferior

(s egme nto torá cico)

Vena cava inferior

(segme nto abdomin al)

Afluentes de la VCI

(s egme nto abdomin al)
A. Porción intrapericárdica

B. Porción extrapericárdica

C. No refiere diferenciación entre segmentos

A. Segmento subhepático a. Segmento Subduodenal

Relaciones: Art. iliaca común derecha

Raíz del mesenterio

b. Segmento Retroduodenopancreático

Relaciones: Duodeno-páncreas

Art. ovárica o testicular derecha

Vena Porta Hepática

Conducto colédoco

Arcos vasculares pancreato-

c. Segmento Supraduodenopancreático

Relaciones: Foramen epiploico

B. Segmento retrohepático

Relaciones: Ligamento coronario

C. No refiere diferenciación entre segmentos

Venas lumbares

Venas frénicas inferiores

Vena testicular u ovárica derecha

Vena renal derecha

Vena renal izquierda

Vena suprarrenal derecha

Venas hepáticas

En caso afirmativo, especifique:
Variantes anatómic as halladas

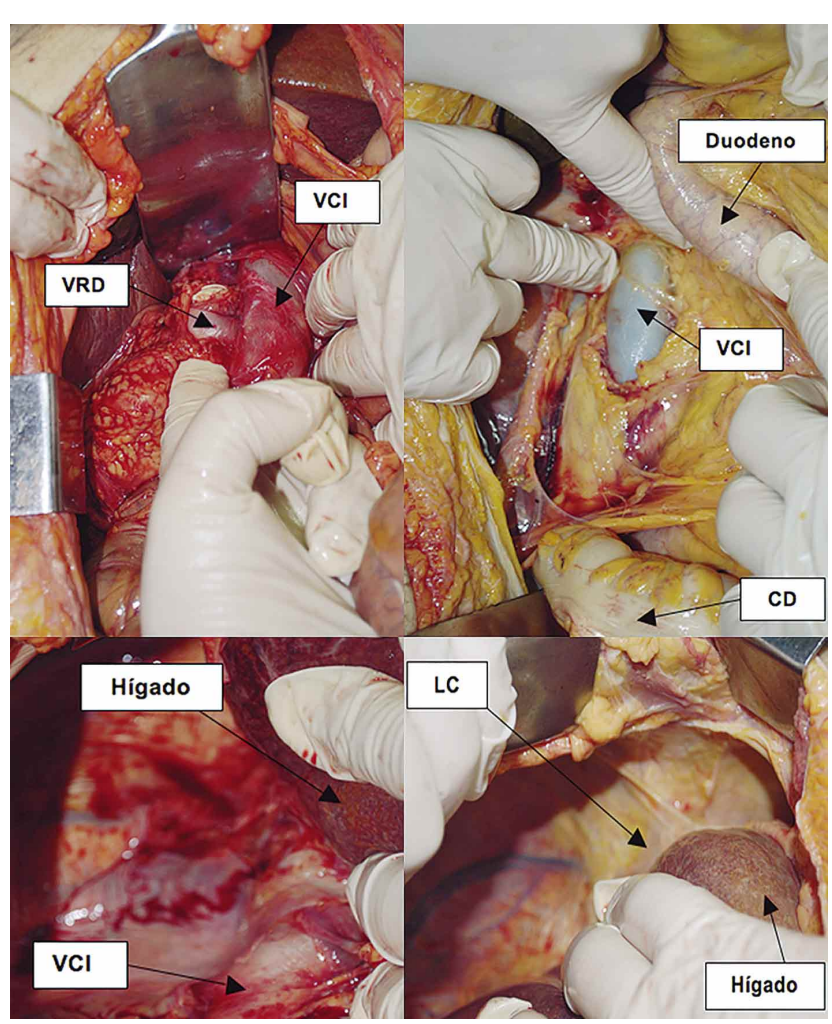

supraduodenopancreático como parte del segmento subhepático. Tres residentes (25\%) no mencionaron los diferentes segmentos de la VCI (abdominal). El segmento retrohepático fue identificado por ocho residentes $(66,66 \%)$ y uno $(8,33 \%)$ lo identificó pero no recordó su nombre.

Respecto a las relaciones, nueve residentes (75\%) identificaron la arteria iliaca común derecha, siete residentes $(58,33 \%)$ a la raíz del mesenterio; ocho residentes (66,66 $\%)$ al duodeno-páncreas; cinco residentes $(41,66 \%)$ a la arteria ovárica o testicular derecha; siete residentes $(58,33$ $\%)$ a la vena porta; siete residentes $(58,33 \%)$ al conducto colédoco; cuatro residentes $(33,33 \%)$ a los arcos vasculares pancreato-duodenales; tres residentes $(25 \%)$ al foramen omental y dos residentes $(16,66 \%)$ al ligamento coronario (Fig. 3).

Respecto a los afluentes, cinco residentes (41,66\%) identificaron las venas lumbares; cuatro residentes $(33,33$ $\%)$ las venas frénicas inferiores; seis residentes (50\%) las

Fig. 2. A-D) Material cadavérico fresco utilizado en la segunda evaluación. $\mathrm{VCI}=$ vena cava inferior; $\mathrm{VRD}=$ vena renal derecha; $\mathrm{LC}=$ ligamento coronario $; \mathrm{CD}=$ colon derecho. 
venas testicular u ovárica derecha; 10 residentes $(83,33 \%)$ la vena renal derecha; 10 residentes $(83,33 \%)$ la vena renal izquierda; seis residentes $(50 \%)$ la vena suprarrenal derecha y ocho residentes $(66,66 \%)$ las venas hepáticas (Fig. 4).

Durante la segunda evaluación la vena iliaca común derecha e izquierda, fueron identificadas por 12 residentes (100 $\%)$. Once residentes $(91,66 \%)$ mencionaron los segmentos de la VCI en intratorácico y abdominal. Quienes identificaron el segmento intratorácico, mencionaron la porción intrapericárdica y la porción extrapericárdica. El segmento abdominal fue identificado por 12 residentes (100\%). De éste segmento, la porción o segmento subduodenal fue identificado como subhepático por dos residentes (16,66\%); y nueve residentes ( $75 \%$ ) mencionaron los segmentos subduodenal, retroduodenopancreático y supraduodenopancreático como parte del segmento subhepático. Un residente $(8,33 \%)$ no mencionó los diferentes segmentos de la VCI (abdominal). El segmento retrohepático fue identificado por 11 residentes $(91,66 \%)$.

Respecto a las relaciones, 12 residentes (100\%) identificaron la arteria iliaca común derecha, nueve residentes $(75 \%)$ a la raíz del mesenterio; 10 residentes $(83,33 \%)$ al duodenopáncreas; ocho residentes $(66,66 \%)$ a la arteria ovárica o testicular derecha; 11 residentes $(91,66 \%)$ a la vena porta; 11 residentes $(91,66 \%)$ al conducto colédoco; seis residentes (50 $\%)$ a los arcos vasculares pancreato-duodenales; ocho residentes $(66,66 \%)$ al foramen omental y siete residentes $(58,33 \%)$ al ligamento coronario (Fig. 5).

Respecto a los afluentes, nueve residentes $(75 \%)$ identificaron las venas lumbares; siete residentes $(58,33 \%)$ las venas frénicas inferiores; 10 residentes $(83,33 \%)$ las venas testicular u ovárica derecha; 12 residentes $(100 \%)$ la vena renal derecha; 12 residentes $(100 \%)$ la vena renal izquierda; 11 residentes $(91,66 \%)$ la vena suprarrenal derecha y 12 residentes (100\%) las venas hepáticas (Fig. 6).

\section{DISCUSIÓN}

Etimológicamente la palabra "cava" deriva del latín cavus que significa hueco y anatómicamente la vena cava inferior consiste en un tronco común retroperitoneal al cual le van a llegar las venas que drenan el abdomen, la pelvis y los miembros inferiores (Motta Ramírez et al., 2010). Se origina por delante del disco intervertebral que separa la cuarta de la quinta vértebra lumbar, a uno o dos centímetros por debajo de la bifurcación aórtica, a partir de las venas ilíacas primitivas derecha e izquierda (Latarjet \& Ruiz Liard; Drake et al., 2010; Moore $e t$ al.). A lo largo de su trayecto ascendente se ubica anterior y a la derecha de la columna vertebral para ubicarse

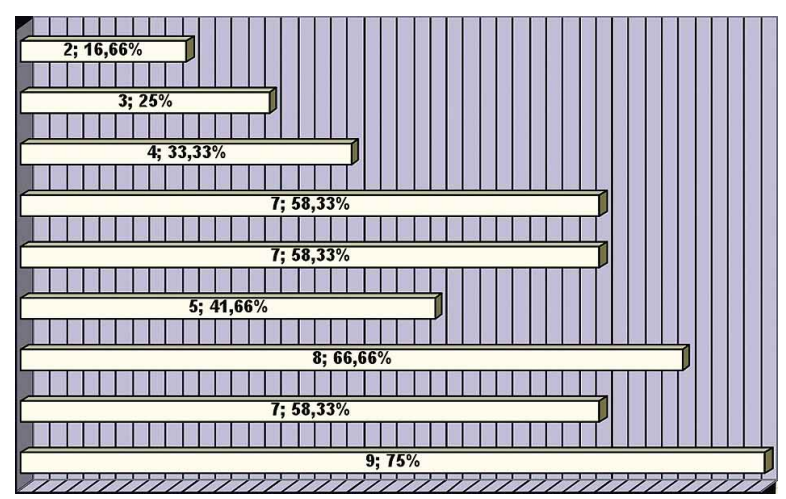

Fig. 3. Relaciones identificadas durante la primera evaluación con material cadavérico formalizado al $10 \%$.

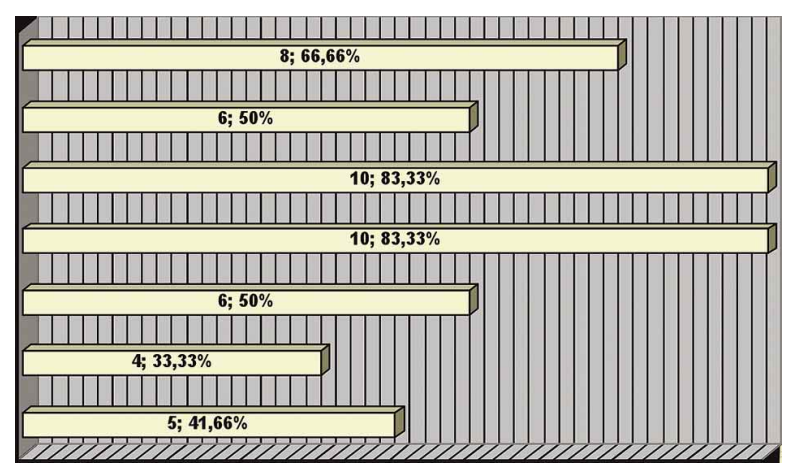

Fig. 4. Afluentes identificados durante la primera evaluación con material cadavérico formalizado al $10 \%$.

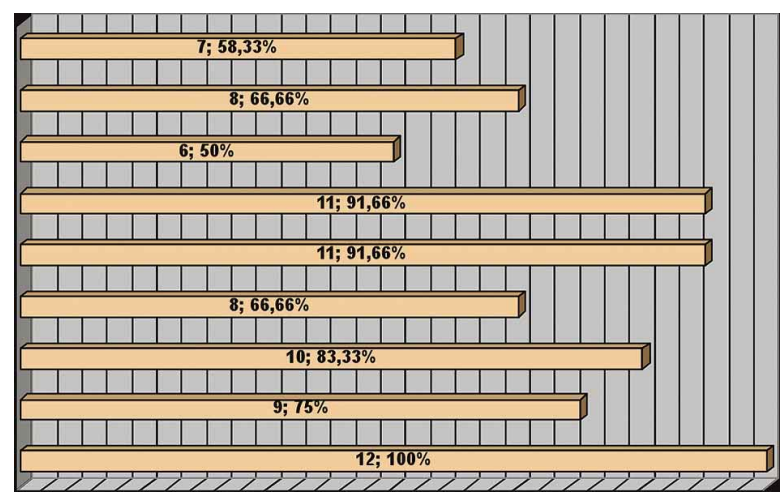

Fig. 5. Relaciones identificadas durante la segunda evaluación con material cadavérico fresco.

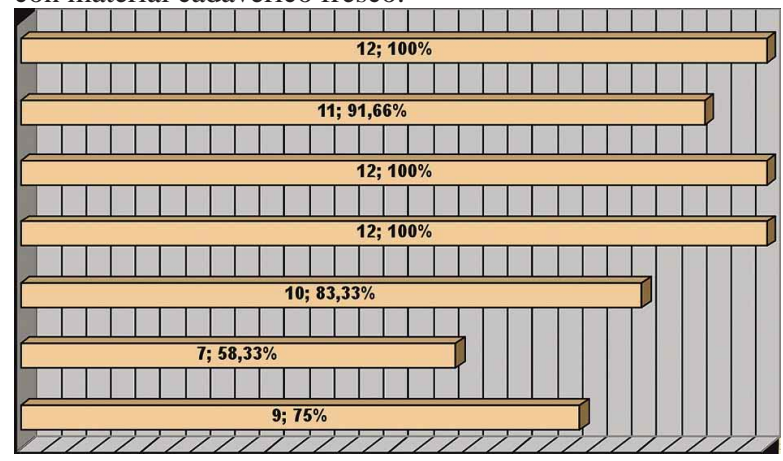

Fig. 6. Afluentes identificados durante la segunda evaluación con material cadavérico fresco. 
posterior al hígado a nivel de la primera vértebra lumbar y duodécima vértebra dorsal, atraviesa el centro frénico del diafragma por un orificio que le es propio limitado hacia atrás por el VI cartílago costal derecho entre las hojas media y derecha del centro tendinoso acompañado del nervio frénico derecho. Ingresa de este modo a la cavidad torácica a nivel de la Octava vertebra torácica, para finalizar su recorrido perforando el pericardio y desembocando en la parte posterior e inferior de la aurícula derecha a nivel de la novena vertebra dorsal. Su longitud varía entre de 22$25 \mathrm{~cm}$, dependiendo de la talla de la persona, con un diámetro medio de $20-22 \mathrm{~mm}$ en su origen y $30-32 \mathrm{~mm}$ en su terminación; va aumentando sus dimensiones a medida que asciende, presentando dos ensanchamientos, uno a nivel de la desembocadura de las venas renales y otro a nivel de la desembocadura de las venas hepáticas (Rouviere \& Delmas; Moore et al., Pró). Recibe como venas tributarias a las venas lumbares, las venas frénicas inferiores, las venas iliacas internas y externas, la vena renal derecha e izquierda, la vena gonadal derecha (ovárica o espermática) y la suprarrenal derecha. Las venas suprarrenal y gonadal izquierdas drenan indirectamente a través de la vena renal izquierda. Las venas hepáticas traen el drenaje de la vena porta desembocando en la vena cava inferior (Testut \& Latarjet; Latarjet \& Ruiz Liard; Moore et al.).

Así, tradicionalmente se describen anatómicamente porciones de la vena cava inferior para considerar las relaciones de la misma; así, autores clásicos como Testut \& Latarjet, Latarjet \& Ruiz Liard, Drake et al., Moore et al. y Rouviere \& Delmas describen una porción o segmento intraabdominal, otro a nivel diafragmático y otro intratorácico. Testut \& Latarjet, Latarjet \& Ruiz Liard y Rouviere \& Delmas, describen dos porciones intratorácicas a saber, extrapericárdica e intrapericárdica. Testut \& Latarjet, Drake et al., Moore et al. y Rouviere \& Delmas no describen segmentos de la porción intraabdominal; en cambio, Latarjet \& Ruiz Liard y Pró describen un segmento subhepático y otro retrohepático; y dentro del segmento subhepático se incluyen: segmento subduodenal, segmento retroduodenopancreático y segmento supraduodeno-pancreático.

En la actualidad, uno de los mayores dilemas al que se enfrenta el cirujano general es cómo reparar lesiones vasculares por trauma en medio de una contaminación masiva, evitando la infección (Feliciano; Feliciano et al.; Asencio et al.). Debe entenderse que debido a su localización retroperitoneal y a la proximidad de otros órganos, los vasos abdominales rara vez son lesionados aisladamente, por lo que la lesión multiorgánica es más la regla que la excepción (Asencio et al.).
El adecuado conocimiento anatómico, brinda un apropiado índice de sospecha de lesión. De modo que se debe concientizar al cirujano de que cualquier herida penetrante en el dorso, desde la línea mamilar hasta los muslos, significa un elevado riesgo de lesión vascular, las cuales son altamente letales. Además, las lesiones penetrantes en la línea media se asocian frecuentemente con lesión aórtica o de la cava; y las heridas periumbilicales pueden afectar asimismo a la aorta y a la bifurcación de la cava. Las heridas por arma de fuego que atraviesan la cavidad abdominal o la pelvis también poseen alto riesgo de lesión de estructuras vasculares abdominales. Por otro lado, cuando ocurren en el hipocondrio derecho, la sospecha de lesión se debe dirigir, anatómicamente, hacia la aorta, la cava y el eje portal (Asencio \& Lejarraga, 2000; Asencio et al.).

La vena cava puede estar libre en su totalidad pero muchas veces el cirujano puede encontrarse con procesos que entorpecen o imposibilitan su adecuada visualización en la cirugía abdominal. Así, como también se pueden presentar diferentes complicaciones como laceración de trayectos venosos, o arrancamiento de ramas lumbares de la vena cava, entre otras (Martinez, 2004); incluso se puede encontrar con diferentes variantes anatómicas de este gran vaso y sus ramas que están descriptas en la bibliografía (VCI izquierda; doble VCI; vena ácigos como continuación de la VCI; ausencia de VCI con preservación del segmento suprahepático; doble VCI con vena renal izquierda retroaórtica y continuación ácigos; vena renal retroaórtica; vena renal circumaórtica; doble VCI con vena renal derecha retroaórtica y continuación hemiácigos); las cuales deben tenerse en cuenta a la hora de enfrentar una cirugía abdominal en la que se deba acceder al retroperitoneo o en la que se pueda encontrar con probable lesión del sistema de la VCI, a modo de evitar una catástrofe intraoperatoria por desconocimiento anatómico (Chuang et al., 1974; CastroIglesias et al., 2010; Motta Ramírez et al.; Morató et al., 2012; Algieri et al., 2013; Rodríguez et al., 2013).

\section{CONCLUSIÓN}

En el manejo y tratamiento de lesiones de vena cava inferior, los mejores resultados se obtienen cuando el cirujano cuenta, en primera medida, con el conocimiento anatómico de este gran vaso, así como de las estructuras que se encuentran en relación con el mismo, además de la experiencia y el equipamiento quirúrgico necesario para encarar este tipo de lesiones. Por lo que la observación y disección anatómica en material cadavérico durante la formación del cirujano general, brinda un importante método de enseñanza, capacitación y entrenamiento hacia el reconocimiento 
de las diferentes estructuras anatómicas de la región, pudiendo luego ser aplicado en cirugía. La metodología de supervisión y evaluación mediante listas de verificación, por médicos especialistas, es una opción que podría integrarse a los programas de formación para mejorar el proceso de educación.

ALGIERI, R. D.; FERRANTE, M. S.; FLORES, C.; PAGLILLA, P.; INSFRAN, H. \& OTTONE, N. E. Evaluation of anatomical and surgical understanding of the inferior vena cava system by general surgery interns. Int. J. Morphol., 34(2):424-430, 2016.

SUMMARY: Injuries in the inferior vena cava (IVC) have been described as the most common vascular abdominal injury found in trauma. The injury may present between the suprarenal and the infrarenal; in the case of retrohepatic injuries, it is associated with a high mortality rate. In a penetrating wound, high mortality rates are reported. Up to $50 \%$ of the individuals who experience these injuries die before getting to the hospital, and the mortality rate among those who get to a healthcare facility alive, ranges from 20 to $57 \%$. The study involved the evaluation of 12 trainee surgeons concerning anatomical understanding of the inferior vena cava system and related areas, using corpses subject to $10 \%$ formol via checklists (April 2014). After a training period with anatomical dissection of 10 corpses subject to $10 \%$ formol (May 2014-August 2014), the general surgery interns were reassessed via checklists using fresh corpses fresco (September 2014). During the first evaluation, the right and left common iliac vein were identified by 8 (66.66 \%) interns: 7 (58.33\%) mentioned the segments of the IVC in the intrathoracic and abdominal area; $6(85.71 \%)$ referred to the intrapericardial portion, and 1 $(14.29 \%)$ mentioned the extrapericardial portion. The subduodenal portion or segment was identified as subhepatic by 7 (58.33\%) interns, and only $2(16.66 \%)$ referred to the subduodenal, retroduodenopancreatic and supraduodenopancreatic segments as part of the subhepatic segment. $3(25 \%)$ failed to mention the various segments of the (abdominal) IVC. The retrohepatic segment was identified by $8(66.66 \%)$ interns. During the second evaluation, the right and left common iliac vein were identified by $12(100 \%)$ interns: $11(91.66$ $\%)$ mentioned the segments of the inferior vena cava in the intrathoracic and abdominal areas; 11 (91.66 \%) interns referred to the intrapericardial and the extrapericardial portions. The subduodenal segment was identified as subhepatic by 2 (16.66 \%) interns, and 9 (75\%) of them mentioned the subduodenal, retroduodenopancreatic and supraduodenopancreatic segments as part of the subhepatic segment. The retrohepatic segment was identified by 11 (91.66 \%) interns. Observation and anatomical dissection of the IVC in corpses when training a general surgeon provides a major teaching and training method to recognize the different anatomical structures of the area, for subsequent its application to surgery. The supervision and evaluation methodology consisting of the use of checklists by specialist physicians is an option that should be added to training programs in order to improve the educational process. in training.

KEY WORDS: Inferior vena cava; Cadaveric models; Anatomical and surgical knowledge; Surgical training; Surgeons

\section{REFERENCIAS BIBLIOGRÁFICAS}

Algieri, R. D.; Ferrante, M. S.; Lazzarino, C.; Fernández, J. P. \& Ugartemendia, S. Vena renal izquierda con trayecto retroaórtico. Rev. Argent. Anat. Online, 4(2):51-5, 2013.

Asencio, J. A. \& Lejarraga, M. Abdominal Vascular Injury. En: Demetriades, D. \& Asensio, J. A. (Eds.). Trauma Handbook. Austin (TX), Landes Biosciences Co., 2000. pp.356-62.

Asencio, J. A.; Petrone, P.; Kimbrell, B. \& Kuncir, E. Lesiones vasculares abdominales. El desafío del cirujano de trauma. Rev. Colomb. Cir., 22(2):124-34, 2007.

Castro-Iglesias, A. M.; Díaz-Bermúdez, J.; Gago-Ferreiro, C. \& Noya-Castro, A. Vena cava inferior doble. Actas Urol. Esp., 34(9):823-4, 2010.

Chuang, V. P.; Mena, C. E. \& Hoskins, P. A. Congenital anomalies of the inferior vena cava. Review of embryogenesis and presentation of a simplified classification. Br. J. Radiol., 47(556):206-13, 1974.
Drake, R. L.; Vogl, W. \& Mitchel, A. W. M. Gray. Anatomía para Estudiantes. Barcelona, Elsevier, 2010.

Feliciano, D. V. Abdominal Vessels. En: Ivatury, R. \& Cayten, C. G. (Eds.). The Textbook of Penetrating Trauma. Baltimore (MA), Williams \& Wilkins, 1996. pp.702-16.

Feliciano, D. V.; Burch, J. M. \& Graham, J. M. Abdominal Vascular Injury. En: Mattox, K. L.; Feliciano, D. V. \& Moore, E. E. (Eds.). Trauma. $4^{\text {th }}$ ed. New York, McGraw Hill, 1999. pp.783-805.

Fuentes, J.; Caminos, P.; Montenegro, R.; Chércoles, R. A.; Casaretto, E.; Canga, C. \& Florez Nicolini, F. Traumatismos penetrantes de vena cava inferior: criterios terapéuticos y manejo quirúrgico. Rev. Argent. Cir., 84(1-2):14-21, 2003.

Latarjet, M. \& Ruiz Liard, A. Anatomía Humana. Vol 2. $4^{\mathrm{a}}$ ed. Buenos Aires, Médica Panamericana, 2005. 
ALGIERI, R. D.; FERRANTE, M. S.; FLORES, C.; PAGLILla, P.; INSFRAN, H. \& OTTONE, N. E. Evaluación del conocimiento anátomo-quirúrgico del sistema de la vena cava inferior por médicos residentes de cirugía general. Int. J. Morphol., 34(2):424-430, 2016.

Martinez, J. M. P. Cómo solucionar accidentes intraoperatorios en la vena cava inferior y sus ramas. Rev. Argent. Urol., 69(4):241-4, 2004.

Moore, K. L.; Dalley, A. F. \& Agur, A. M. R. Anatomía con Orientación Clínica. $7^{\mathrm{a}}$ ed. Philadelphia, Lippincott Williams \& Wilkins, 2013.

Morató, L. A. E.; Quiróz, C. O. \& Ramírez, A. J. L. Anomalías congénitas de la vena cava inferior. El rol de la tomografía computada multidetector. An. Radiol. Mex., 11(2):104-13, 2012 .

Motta Ramírez, G. A.; Mundo Gómez, C. \& Ramírez Arias, J. L. La vena cava y sus variantes anatómicas. Rev. Mex. Angiol., 38(1):19-29, 2010

Ponce de León Espíndola, M. \& López Cueto, A. Lesión aislada de vena cava inferior por traumatismo penetrante de abdomen. Rev. Trauma Am. Lat., 1(1):45-8, 2011.

Pró, E. A. Anatomía Clínica. 2a ed. Buenos Aires, Médica Panamericana, 2014.

Rodríguez, A.; Cobeñas, R.; Cruz Gallo, J.; Salamida, A.; Larrañaga, N. \& Kozima, S. Variantes anatómicas vasculares halladas de manera incidental en estudios de tomografía computada. Rev. Argent. Radiol., 77(1):19-25, 2013.

Rouviere, H. \& Delmas, A. Anatomía Humana. Descriptiva, Topográfica y Funcional. Tomo $2.11^{\mathrm{a}} \mathrm{ed}$. Barcelona, Masson, 2005.

Testut, L. \& Latarjet A. Tratado de Anatomía Humana. $8^{\text {a }}$ ed. Barcelona, Salvat, 1943.
Dirección para Correspondencia:

Rubén Daniel Algieri

Especialista en Cirugía General

Jefe de Servicio de Cirugía General del Hospital Aeronáutico

Central.

Prof. Regular Adjunto de Anatomía y Docente Adscripto en Cirugía III Cátedra de Anatomía Humana

Facultad de Medicina

Universidad de Buenos Aires

Paraguay 2155. C.A.B.A.

Buenos Aires

ARGENTINA

Email: rdalgieri08@hotmail.com

Recibido : 14-01-2016

Aceptado: 20-03-2016 